\title{
EXPERIMENTS IN IDENTIFICATION AND CONTROL OF FLEXIBLE-LINK MANIPULATORS ${ }^{1}$
}

\author{
Stephen Yurkovich Anthony P. Tzes Fernando E. Pacheco \\ The Ohio State University \\ Department of Electrical Engineering \\ 2015 Neil Avenue \\ Columbus, Ohio $\{3210$
}

\begin{abstract}
Interest in the study of flexible-link manipulators for space-based applications has risen strongly in recent years. Moreover, numerous experimental results have appeared for the various problems in the modeling, identification and control of such systems. Despite the recent activity throughout the literature for flexible-link manipulator control, relatively little has appeared involving laboratory verification of tuning controllers for realistic flexible-link manipulators which are required to maintain endpoint accuracy while manipulating loads which are possibly unknown and varying, and while undergoing disturbance effects from the environment and in the workspace. This paper reports on an ongoing effort in these areas for endpoint position control of flexible-link manipulators, with laboratory setups consisting of one and two-link manipulators.
\end{abstract}

\section{Introduction}

Efforts in the modeling and control of flexible-link manipulators have been motivated by the foreseen demand for lightweight, accurate, high-speed robots in space telerobotcs and several other applications. Presently, studies in these areas have reached a fairly high level of malurity, due primarily to numerous works in the last four years from both an analytical viewpoint and, to a lesser extent, experimental viewpoint. Analytical studies in modeling flexible-link robots abound, and are in fact too numerous to cite here; References $[1,2]$ serve as excellent summaries of existing works in Hexible manipulator modeling. Equally numerous are the various approaches which have appeared in the literature for controller design schemes. The greatest number of these works have dealt in simulation studies only, and some have developed quite elabcrate and complex control schemes.

On the other hand, several successful labotatory setups have demonstrated the effectiveness of relatively simple algorithns for flexible manipulator control. While most experimental studies have focused on single-link manipulators, or multi-link manipulators with a single flexible link, such setups have served as valuable testberls for modeling. system identification and controller design. Some of the more visible experimental efforts have been the work of Yurkovich ot al. $[3,4,5,6]$, Schmitz, Rovner, $\epsilon$ al. $[7.8,9]$, and Book t al. $[10,11]$, among others. In $[4]$ the the of measurements from a linear accelerometes in vibration compensation of the robot endpoint was shown to be extremely successful, proving the concept of acceleration feedback for flexille-link manipulator control. The

\footnotetext{
${ }^{1}$ Supported in part by the Natinnal Aeronatics \& Space Administration under NASA Grant NAG-1-720.
} 
use of acceleration feedback has intuitive appeal from an engineering design viewpoint, due to ease of implementation, relatively low cost, and advantages of structure-mounted sensing.

Despite this recent activity, relatively little has appeared involving laboralory verification of tuning controllers for realistic flexible link manipulators which are required to maintain endpoint accuracy while manipulating loads which are possibly unknown and varying. This paper discusses several techniques for flexible-link systems, and presents experimental results in system identification and control of a one-link Hexible manipulator carrying an unknown, varying payload.

\section{Problem Description}

T'wo laboratory setups are currently utilized in the Flexible Structures Facility at Ohio State (Department of Electrical Engincering) $[12,13]$. The one link system is the subject of the experimental results reported in this paper and is described in detail below. The identification and control techniques described are, however, currently being investigated primarily for the second system which consists of two flexible links situated in the horizontal plane. The first link, which is driven by a $3.4 \mathrm{ft} / \mathrm{b}$ direct drive servomotor, is made of aluminum, 0.75 meter in length and 0.125 inches in width. Mounted at the endpoint is a $1.5 \mathrm{in}-\mathrm{lb}$ geared servomotor to actuate the smaller (0.5 meter aluminum) second link, 0.0625 inches in width. Both links are therefore very flexible, and the setup lends itself to complicated modeling, identification and control problems. A VME Bus Motorola $68020 / 68881$ system, with 16 channels of $A / D$ and four channels of $D / A$, is used as the control computer. Results of experimentation with this apparatus are forthcoming.

\section{A. One-Link Setup}

The flexible-link manipulator arm of this study is a beam made of $\frac{1}{16}$ inch 6061-T6 alunumum, one meter in length and $10 \mathrm{~cm}$ in height. The arm is counterbalanced about the motor axis with a rigid aluminum attachment $38 \mathrm{~cm}$ in length. An electromagnet device is mounted on the manipulator endpoint to facilitate experimentation with different payloads. Actuation at the hub is accomplished by a direct drive de motor with rated stall torque at $680 \mathrm{oz}$-in. The two sensors for use in feedback control are the accelerometer, located at the arm endpoint, and the optical shaft encoder located at the hub. The encoder is rated at 3600 pulses per revolution, allowing measurement of the shaft angular position with a resolution of 0.05 degrees. The piezoelectric accelerometer is rated at $\pm 250 \mathrm{~g}$ with a sensitivity of $1.15 \mathrm{mV} / \mathrm{g}$. A linear array line scan camera is used for data recording, reading a light source at the arm endpoint, but is not used in feedback control (results of enclpoint position feedback for this setup were presented in [4]). The computer used in the lata acquisition and control is the DEC MicroVax II.

\section{B. Identification and Control}

The laboratory setup has furnished an excellent testbed for investigation of many ideas in the areas of identification and control, several of which are described in the sections to follow. Specifically, methodologies under study have included

- Position feedback, fixed controller designs;

- Acceleration feedback, fixed controller designs:

- Eigenstructure realization algorithms for identification;

- Auto-tuning control designs with identification, time domain:

- Auto-tuning control designs with identification, frequency domain;

- Input shaping with acceleration feedback;

- Learning schemes; 
- Time optimal slewing controllers.

A characteristic of flexible-link manipulators situated in the horizontal plane is that the morlal frequencies are reduced when a payload is added. Motivated by this and the fact that a fixed controller will not perform well over a range of payloads, the idea pursued in much of the techniques listed above is to tune a nominal control configuration according to the changing characteristics of the arm. As an illustration, consider the nominal case, that is, when no load is carried by the arm. This nominal control scheme utilizes endpoint acceleration through a static feedback gain, with shaft position in a separate static gain feedback loop. We note that more complex schemes have been investigated, such as linear quadratic regulator theory, or inclusion of dynamics in the compensation network, but the primary objective was to attain good performance with the simplest possible control technique. This acceleration feedback control scheme is very robust to disturbance effects (can maintain endpoint position even when the arm is jolted), but, as might be expected, cannot perform nearly as well for significant payload variation. This effect is verified experimentally by having the arm carry a payload weighing $0.67 \mathrm{lbs}$, which is approximately $63 \%$ of the weight of the arm itself. Figure 1 shows the results of this exercise, where the same controller gains utilized in the nominal, no-load case (dashed curve) are employed for the case with payload; the response is to a commanded input which basically demands that the arm endpoint follow a square wave reference. Attempts at designing fixed controllers with more complicated dynamics were only slightly more successful. Indeed, the large overshoot could be avoided, and endpoint position accuracy maintained, if the control gains were tuned appropriately. For purposes of comparison, Figure 2 offers the open loop response which illustrates the flexibility of the arm, even for a relatively small slew angle of only about $15^{\circ}$.

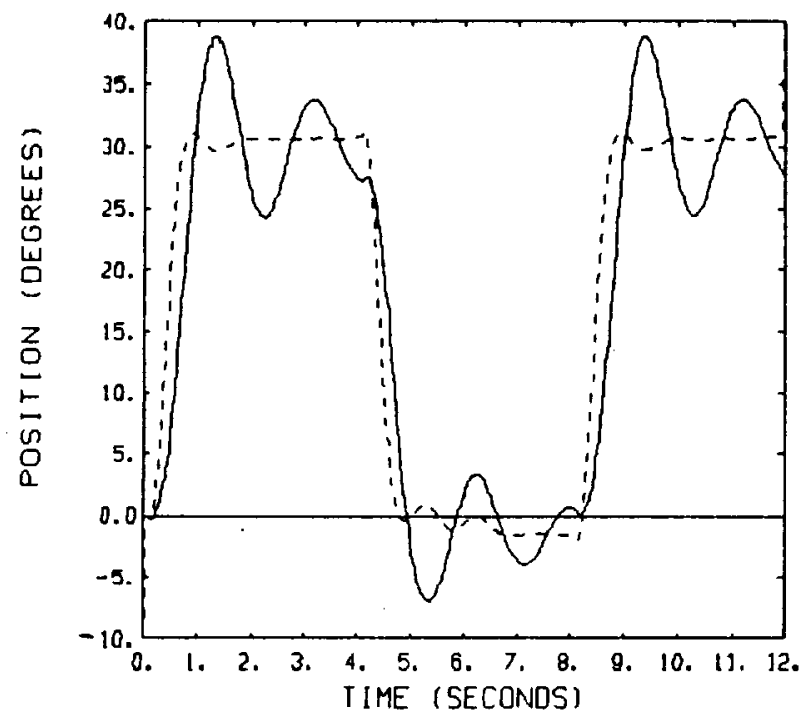

Figure 1: Effect of Payload

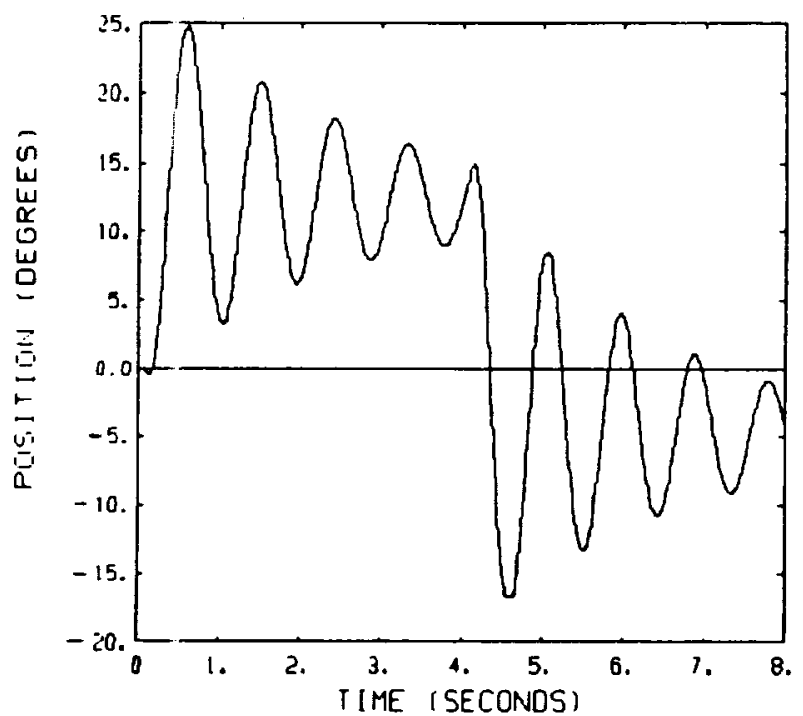

Figure 2: Open Loop (no control)

\section{Time Domain Auto-Tuning Control}

\section{A. Identification}

Since the control objective we consider in this problem involves vibration suppression afterthe manipulator has undergone the nonlinear slew maneuver, a reasonable choice of model structure 
amenable to control design for the input to shaft angle and the input to endpoint acceleration transfer functions (filter) is the Autoregressive Moving Average (ARMA) model. Within this setting, consider

$$
y(k)=\phi^{T}(k) \theta+w(k),
$$

where $\theta$ is the vector of filter coefficients, $w(k)$ is stationary, zero mean process noise, and $\phi^{T}(k)=$ $[y(k-1), \ldots, y(k-n), u(k-d-1), \ldots, u(k-d-n)]$ is the regression vector, for $y(k)$ and $u(k)$ the system output and input, respectively, and $d$ is the inherent delay (in sampling time multiples) between the commanded input (for hub torque actuation) and the response seen in the shalt angle or, more noticeably, in the endpoint acceleration.

For filter parameter updates we limit our discussion here to the least squares and recusive least squares (RLS) algorithms. Both techniques are based on computation of the optimal value of the parameter vector $\theta$ based on minimization of a scalar loss function of the squared equation error. That is, the well-known non-recursive solution to this procedure for the model (1) is given by

$$
\hat{\theta}==M \Phi^{T} y,
$$

where the information matrix $M=\left[\Phi^{T} \Phi\right]^{-1}$ is constructed from the data $\phi$, and $\hat{\theta}$ is the estimate of $\theta$. Although computationally fast, the amount of data needed for reliable parameter convergence marle RLS only slightly faster than a recursive implementation of an information matrix form of standard least squares (LS). We have therefore chosen to use such a form of LS which tended to give better estimator performance, traded off against computational burden. In the robolic system application we consider here, payload changes are of a discrete nature at a given point in time, implying that there is no requirement for remembering previous load characteristics. Best results were obtained, then, using a weighted version of the non-recursive expression

$$
M^{-1}(k+1) \hat{\theta}\left(k^{2}+1\right)=\Phi^{T}(k+1) y(k+1)
$$

with recursive data information updates according to

$$
\begin{aligned}
M^{-1}(k+1) & =\lambda(k) M^{-1}(k)+\phi(k+1) \phi^{T}(k+1) \\
\Phi^{T}(k+1) y(k+1) & =\lambda(k) \Phi^{T}(k) y(k)+\phi(k+1) y(k+1) .
\end{aligned}
$$

In the above, the weight $\lambda(k)$ is the forgetting factor, and for these applications typically took a value in the range $0.96-0.99$.

\section{B. PID Tuning Controller}

The concept of automatic tuning for a proporional-integral-derivative (PID) control law has been the subject of recent investigations [14], and in fact has been utilized for many years in flight control systems. Motivation for auto-tuning schemes lies in the fact that often times PID controllers are difficult to tune manually, particularly when a high level of precision is important.

The discrete version of the ideal analog PID controller is given by

$$
u(k)=K_{P \epsilon}(k)+\frac{T}{K_{I}} \sum_{i=0}^{k-1} \epsilon(i)+\frac{K_{D}}{T}[\epsilon(k)-\epsilon(k-1)],
$$

where $T$ is the sampling time, $u(k)$ is the control input, $\epsilon(k)$ is the deviation between the controlled signal and a desired reference, and $K_{P}, K_{I}, K_{D}$ represent the proportional, integral, and derivative 
gains, respectively. Generation of a recursive expression for the control input follows easily from (6) as

$$
u(k)-u(k-1)=b_{o} \epsilon(k)+b_{1} c(k-1)+b_{2} \epsilon(k-2) .
$$

At this point several options are available for choice of the partmeters $\left[b_{o}, b_{1}, b_{2}\right]$, such as classical pole placement or pole cancellation design. However, for our problem it may not always be apparent a priori what the desired closed loop poles should be, since their choice may depend on the effect of payload variation. For example, with a larger payload a slower slew maneuver may be required. For this reason we choose the PID parameters via an optimization of a performance criterion which is time varying and which weights the control deviation and the endpoint acceleration.

For the manipulator system of this study it was determined that a performance index which gave adequate results takes the form

$$
\left.J(k)=\sum_{k=1}^{q}\left[k \epsilon_{s}^{2}(k)+50000(\Delta u)\right)^{2}+6 k^{2} \alpha^{2}(k)\right],
$$

where $e_{s}(k)$ represents shaft position error, $\Delta u=u(k)-u(k-1)$, and $\alpha(k)$ is the endpoint acceleration. Notice that this index penalizes endpoint deflections more heavily as time increases, for the following reasoning. In general, when endpoint movement is minimal, the shaft position error term is larger than the acceleration term. Moreover, for a given selection of PID parameters the shaft position error remains virtually the same when a payload is added. However, the endpoint acceleration is noticeably reduced with payload and the relative weight of the square of the acceleration drops. For this system it was observed that endpoint oscillations continue for a relatively long period when a payload is added; this accounts for the $k^{2}$ factor in (8). Thus, minimization of $(8)$ reduces the duration of oscillation. A period of 100 samples $(T=30 \mathrm{~ms})$ was found to be an adequate interval over which to evaluate the performance index. It is straightforward to derive the relationship between the ARMA model representation (1), the cost criterion (8), and the PID parameterization (6) [5].

The controller design now reduces to computing (8) and carrying out an optimization over possible PID parameters. It is quite obvious, and easily verifiable via experimental tests, that one way to reduce vibrations at the manipulator endpoint when a loarl is added is to reduce the slew rate. This of course is viable only to a degree since our objective is to attempt to slew as fast as possible with the best possible performance.

Several combinations of proportional, PI, PD, or full PID designs in either or both feedback loops are possible [5]. Here we consider the case for gain arljustment within each feedback path (shaft angle gain and endpoint acceleration gain). The effectiveness of this identification/control scheme is illustrated in Figure 3 for the following profile. First, the arm without payload undergoes a $25^{\circ}$ slew with no control applied (open loop); large oscillations are apparent in this first phase. The gains are then tuned and the arm undergoes a further $20^{\circ}$ slew in the came direction, then reverses direction for a $45^{\circ}$ slew. During this plase the control has been extremely effective in endpoint vibration compensation. In the next phase a 0.415 pound payload is adiled and the arm undergoes a $20^{\circ}$ slew; after five seconds (allowing for damping of deflections) tuning is performed. In the same direction, the arm is then slewed an additional $25^{\circ}$, and in five second the direction is reversed to complete the profile with a $45^{\circ}$ slew. The two points at which controller tuning was performed are marked on the plot; the total time span for identification and tuning, given the limitations of the laboratory computer, is anywhere from 15 to 25 seconds depending upon the number of parameters tuned in the optimization. For this reason, the time axis in Figure 3 is not marked, but the time period between slew commands is nominally 5 seconds. 


\section{Frequency Domain Auto-Tuning Control}

\section{A. Identification}

An alternative to time domain methods for adaptive filtering is the use of techniques based in the frequency domain $[15,16]$. Frequency domain adaptive filters enjoy several advantages over their time domain counterparts, including reduced computation and a fast rate of convergence. A disadvantage of methods which identify system frequency response, however, is that auto tuning (on-line) contrul design is often at best ad hoc.

As an illustration of one of the major shortcomings of the RLS method, consider the case of identifying the tip acceleration of the one--link apparatus, using zero mean white noise as input. $A$ typical characteristic of time domain methods is the requirement for a persistently exciting input during the identification starting process. This was the case, for example, in [8] where after a significant amount of data was gathered the identifier was turned on and the estimated parameters converged "fast" to the actual ones. That is, although the convergence of RLS is superior to most other time-identification methorls, a large amount of iterations is required for convergence to the artual parameters. Shown in Figure 4, the estimaled transfer function spectrum of the one-link manipulator is plotted for the cases of $64,128,256$, and 512 iterations after the start-up of the identification process (30 $\mathrm{ms}$ sampling). In all the cases a Butterworth filter of 6 th order with a cutuff at $48_{\text {sec }}^{\text {rad }}$ was used to prefilter the data, the order of the estimated ARMA system was 5 (these values were found to produce the best results $[17])$, and all the initial estimated parameters were set to zero. RLS can predict the first mode (at approximately $1 \mathrm{II} z$ ) only after 512 iterations, where the corresponding peak begins to appear. During convergence the estimated poles and zeros of the system were far away from the actual ones. In the case of an abrupt change of the carried payload for the manipulator under consideration, the RLS algorithm needed a significant amount of time to converge to the new system parameters. This characteristic was noticed for the identification and control experiments described in the preceding section, and may not be satisfactory if the control law update scheme is required to respond quickly.

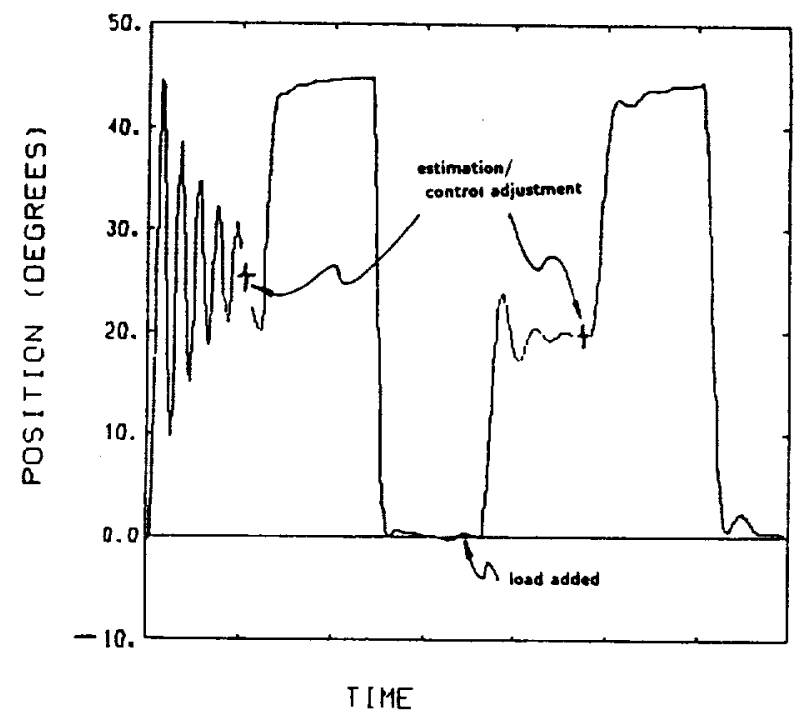

Figure 3: Time Domain Tuning Scheme

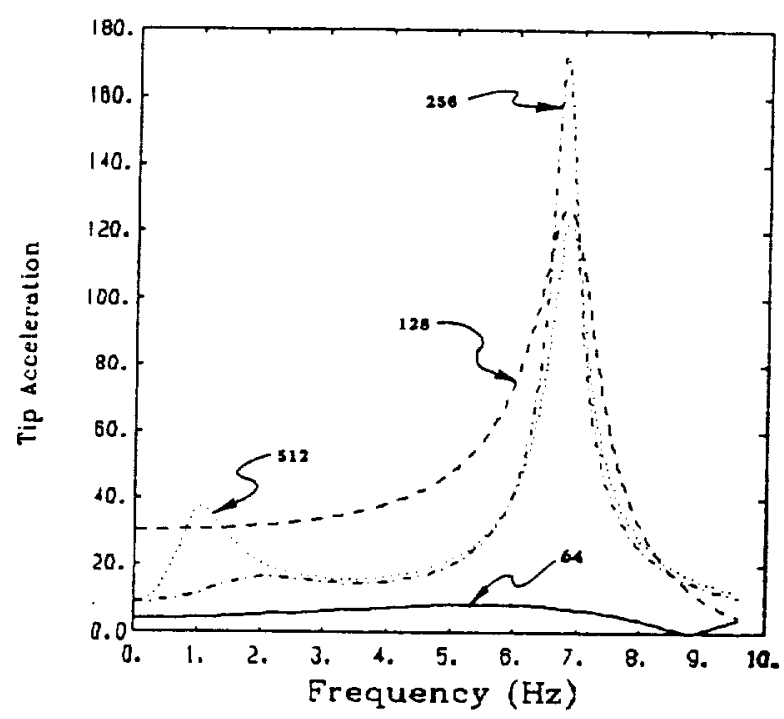

Figure 4: RLS Convergence

By contrast, for on-line filter (transfer function) update, wilh frequency domain methods the system input signal is transformed to the frequency domain before adaptive filtering is applied. The 
simplest frequency-domain adaptive filter is one in which the input signal $u(n)$ and ontput $y(n)$ are accumulated into buffer memories to form $N$-point data blocks. These hlocks are then transformed by $N$-point Fast Fourier Transforms (FFTs) to their equivalent frequency transformed blocks $U, Y$ at the $k^{\text {th }}$ time instant.

A simple yet effective representation for transfer function identification is the Empirical Transfer Function Estimate (ETFE) [18,19]. A non-recursive updating scheme for the transfer function in the frequency domain, at a given time $k$, is utilized in the manner

$$
H_{i}(k)=\frac{Y_{i}(k)}{U_{i}(k)} \quad, \quad H_{N-i}(k)=H_{i}^{*}(k) \quad,
$$

for $i \in\left\{0 \leq i \leq \frac{N}{2}, U(i) \neq 0\right\}$, where $i$ corresponds to the $i^{\text {th }}$ bin in the frequency clomain, and $H_{i}^{*}(k)$ is the complex conjugate of $H_{2}(k)$. Notice that the $H_{i}(k)$ 's can be updated every $l$ samples, where $1 \leq l<N$. The main properties of this technique are that: 1) The variance in the estimation is equal to the signal-to-noise ratio at the frequency under consideration; 2) Estimates at different frequencies are uncorrelated (asymptotically as $k \rightarrow \infty$ ).

A recursive implementation of this idea is possible via the Time-varying Transfer Function Estimation (TTFE) method [20]. The 'TTFF technique can be used to reduce the variance of the estimated frequency response through two distinguishing characteristics. First, the adjacent frequency bins $H_{i}(k), H_{j}(k)$ from Equation $(9)$ are correlated through the relation

$$
H_{i}(k)=\frac{\sum_{j=\left(i-\Delta_{i}\right) \bmod N}^{\left(i+\Delta_{i}\right) \bmod N} \varepsilon_{j}^{i} H_{j}(k)}{\sum_{j=\left(i-\Delta_{i}\right) \bmod N}^{\left(i+\Delta_{i}\right) \bmod N} \varepsilon_{j}^{i}}
$$

which indicates that the estimate $H_{i}(k)$ is related to all the arljacent frequencies within a modulus $\Delta_{i}$ with a corresponding weight $\varepsilon_{j}^{i}$ for the frequency point (bin) $\omega_{j}$. Notice that the case $\Delta_{i}=0$ corresponds to a frequency windowing technique [21] used to reduce the bias and variance of the estimated transfer function. Moreover, the case $\Delta_{i}=\Delta$ for all $i$ and $\varepsilon_{j}^{i}=\varepsilon^{i}=\Phi_{i}(u)$ (where $\Phi_{i}(u)$ is the input spectral density) corresponds to the Blackman-Tukey Procelure [21] for smoothing the estimated transfer function. Therefore, based on this relation the estimated transfer function is a "smoothed" version of the one obtained from ETFE.

The second distinguishing feature of TTFE is that the frequency hin $H_{i}(k)$ is related to the $H_{i}(k-1), \ldots, H_{i}\left(k-\beta_{i}\right)$ bins of the hybrid time-frequency domain through

$$
H_{i}(k)=\mathcal{X}\left[\left(H_{i}(k-1), \ldots, H_{i}\left(k-\beta_{i}\right)\right]\right.
$$

where the function $\mathcal{X}$ may be implemented with RLS for a finite impulse response model $Y_{i}(k)=$ $\sum_{j=1}^{\beta_{i}} H_{i}(k-j) U_{i}(k-j)$. In case of a sudden change of system dynamics, this recursion results in a smooth transient from the old transfer function to the new one, representing a substantial difference when compared to the nonrecursive ETFE technique which suffers a less smooth transition due to the assumption of orthogonalized input-output data blocks. The computational complexity of TTFE is reasonable and can be decreased by assuming that the frequency bins $H_{i}(k), H_{j}(k)$ for the same time instant $k$ are uncorrelated $\left(\Delta_{i}=0\right)$, over a time period of $\gamma$ samples, where $\gamma$ corresponds to the updated interval for the adaptation algorithm.

\section{B. Controller Tuning}

The critical information for control purposes sought by frequency romain methods is the location of poles and zeroes of the transfer function. These locations correspond to the peaks and the valleys 
of the estimated magnitude response. Due to the lightly damped nature of the manipulator these locations are easily recognizable with the TTFE technique, even with signal-to-noise ratios up to $15 d B[1 T]$.

In light of the above discussion on convergence of the parameter estimation, the performance of the I'TFE approach in estimating the first modal frequency of the system is demonstrated via experiment. Figure 5 depicts the endpoint acceleration while the arm is slewed through angles of $30^{\circ}$ in a square-wave (shaft angle position) reference ( 8 second period). The arm carries no payload initially, and the ideal frequency is ahout $1 \mathrm{~Hz}$, indicated by the solid curve. At 8 seconds a $0.485 \mathrm{lb}$ payload ( $46 \%$ of arm weight) is added, and then removed at 16 seconds, resulting in a change in ideal frequency due to the change in payload. Frequency estimates from the TTFE scheme (dashed curve) proved to be adequate, in terms of speed of convergence as well as accuracy, for good controller performance.

Several algorithms for control design using the identified frequency response directly have been implemented for the experimental setup, including a frequency weighted quadratic regulator design [20]. Here we describe results of a design in which the control structure is set within a scheduling framework comprised of two feedback loops: one in which the endpoint acceleration is used as input to a control law, and the other in which the motor shaft angle is input to a separate control law. These two loops are then summed to give a commanded motor input voltage. Motivated by the desire to achieve endpoint position accuracy while maintaining a relatively straightforward implementation structure, simple proportional schemes make up the above-mentioned control laws in the separate loops; it is the individual proportional gains which are scheduled as correlated to frequency domain information over a wide range of payloads. The scheme discussed in the previous section (PID tuning using (8)) was used to establish a "look-up" table for various payloads corresponding to the first modal frequency of the arm. That is, the fundamental frequency (first mode) was found using FFT analysis; because these calculations are carried out off-line, an ample amount of data can be accumulated for the best possible accuracy. The motivation for using the fundamental frequency as the "pointer" in a look-up lable of scheduled optimal controller parameters is the obvious relationship with the payload. This fact is exploited in the control law by interpolating four such data points (using four different payloads) in construction of a functional relationship, filling out the look-up table, for use in real-time control.

Figure 6 shows results of the scheduling controller using frequency domain estimation; shown is the arm endpoint position as read by the camera. As before, the gross motion control objective is to track a staircase-shaped reference trajectory. At the end of the first and fourth segment, as indicated, the FFT of the endpoint acceleration is computed and the controller is tuned according to the estimated frequency of the first mode. The first segment is performed in the open loop for a slew angle of $8^{\circ}$; the absence of any control effort is evident from the large overshoot. The controller is then adjusted with the estimated frequency-this operation, including FFT calculation, requires less than 0.3 seconds of CPU on the MicroVax computer. In the next two segments the arm is slewed another $32^{\circ}$, then $40^{\circ}$ in the opposite direction; the stabilizing effect of the acceleration feedback controller is evident. A payload of $0.74 \mathrm{lb}$ ( $69 \%$ of total arm weight) is added at the beginning of the fourth segment, as indicated, and the arm is slewed through a commanded angle of $5^{\circ}$. This small level of excitation is sufficient to accurately estimate the first modal frequency, so that the controller is re-tuned to account for the addition of the payload. In the final two segments, the arm is slewed first another $35^{\circ}$, and then $40^{\circ}$ in the opposite direction. Again, the control has adequately compensated for deflections at the endpoint. Since the $5^{\circ}$ slew with payload generates relatively small deflections, to illustrate the effectiveness of the scheduling control the endpoint position for the case of not re-tuning the controller after addition of payload is overlayed in the Figure (dashed line). Similar results were obtained for a variety of payload conditions. 


\section{Conclusion}

This paper has presented a summary of the various idrntification and control techuiques being investigated in the laboratory at Ohio State for flexible-link nanipulator systems. Primary attention in these techniques focuses on the ability of the controller to adjust to changes in dynamics, payload, and working environment. Time domain methods offer identified model structures which are readily available for control design, whereas frequency domain methods, particularly the TTFE approach developed for this application, are more desirable when rapid controller tuning is required.

Only a sample of the results obtained to date were presented here due to space constraints; the interested rearler is encouraged to pursue the listerl references, copies of which are available upon request from the authors.

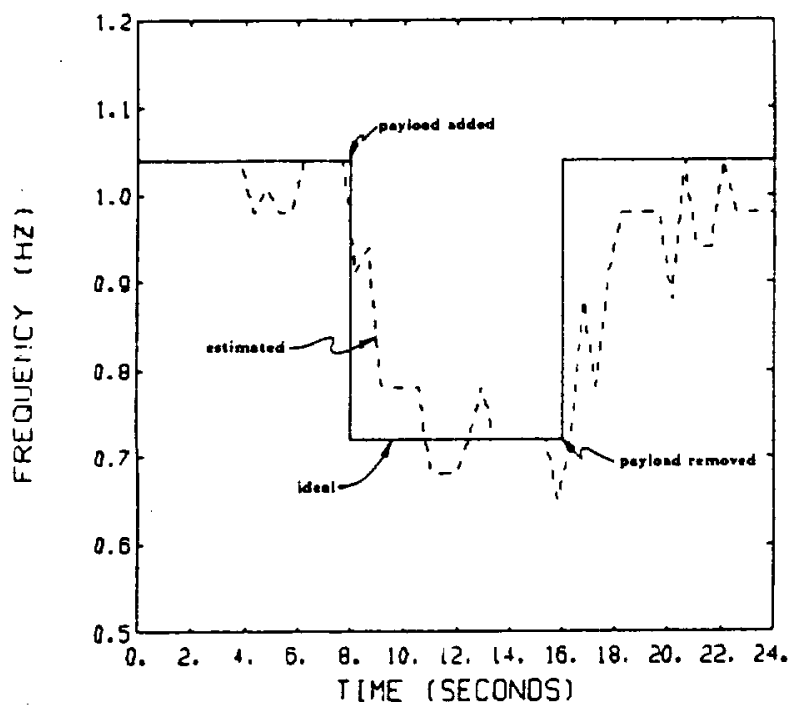

Figure 5: T'TFE Results

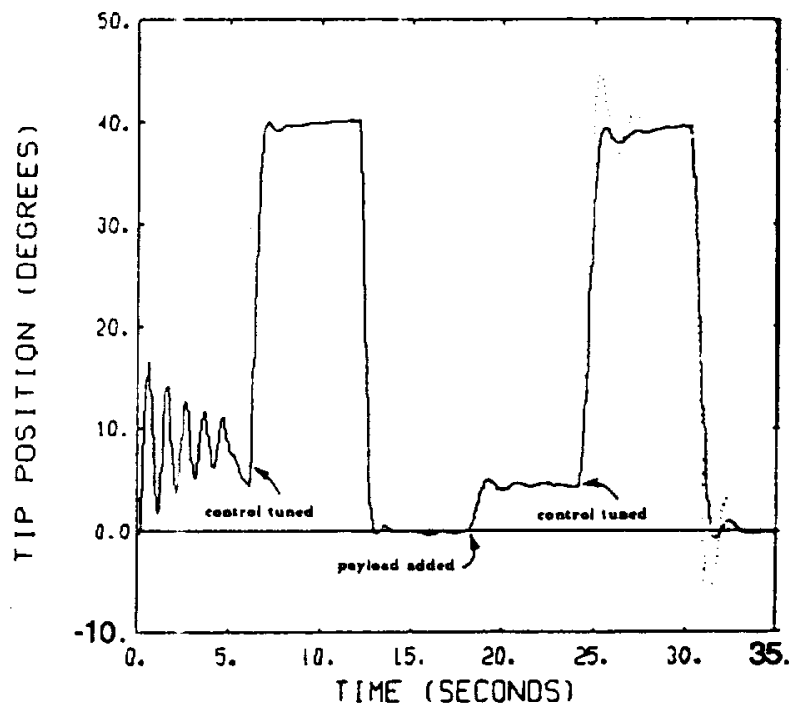

Figure 6: Scheduling Control

\section{References}

[1] X. Ding, T. J. Tarn, and a. K. Bejczy, "A novel approach to the modelling and control of flexible robol arms," in Procecdings of the IEEE Conference on Decision and Control, Austin, TX, December 1988.

[2] F. Barbieri and Ümit Özgüner, "Unconstrained and constrained mode expansions for a flexible slewing link." Trans. ASME, J. Dyn., Mens., and Control, vol. 111, , December 1988.

[3] S. Yurkovich, Ü. Özgüner. A. Tzes. anrl P. Kotnik. "Flexible manipulator cont rol experiments and analysis," in Procefdings of the NASA Telerobotirs Workshop, pp. 279-288, January 1987.

[4] P. Kotnik, S. Yurkovich, and U. Öngüner. "Acceleration feerlhack for control of a flexille manipulator arm." Joumal of Robotic Systems, vol. 5. no. "3, pp. 181-196. June 1988.

[5] S. Yurkovich and F. E. Pacheco, "On controller luninu for a flexible-link manipulator with varying payload," Journal of Robotic Systems. vol. 6, no 3, . June 1989. (to appear).

[6] S. Yurkovich, F. E. Pacheco, and A. P. Tzes. "On-line frequency domain information for control of a llexible-link robot with varving payload," IEEE Transactions on Automatic Control, vol. $\mathrm{AC}-33$, , 1989. (to appear). 
[7] R. H. Canon and E. Schmitz. "Initial experiments on the end-point control of a flexible one-link robot," The International Journal of Robotics Rescarch, vol. 3, no. 3, pp. 62-75, 1984.

[8] D. M. Hovner and R. H. Cannon, "Experiments toward on-line identification and control of a very flexible one-link manipulator," International Journal of Robotics Rescarch, vol. 6, no. 4, pp. 3-19, Winter 1987.

[9] D. M. Rovner and C. F. Franklin, "Experiments in load-adaptive control of a very flexihle one-link manipulator," Automatica, vol. 24, no. 4, pp. 541-548, July 1988.

[10] G. G. Hastings and W. J. Book, "Experiments in optimal cont rol of a flexille arm," in Proceedings of the 1985 American ('ontrol Conference. pp. 728-729, Boston, MA, June 1985.

[11] G. C. Hastings and W. J. Book, "Verification of a linear dynamic model for flexible rohotic manipulators," in Procetdings of the IEEE International Conference on Robotics and Automation, pp. 1024-1029, San Francisco, ('A, April 1986.

[12] Ü. Özüner, S. Yurkovich, I. Martin, and P. Kotnik, "A laboratory facility for flexible structure control experiments," IEEE C'ontrol Systems Magazine, vol. 8, no. 4, , August 1988.

[13] S. Yurkovich and Ümit Özgüner, "Recent developments in the OSU Flexible Structure Control Laboratory," in Proceedings of the Seventh VPI \& AlAA Symposium on Dynamics and Control of Large Structures, Blacksburg, VA, May 1989. (to appear).

[14] F. Radke and R. Isermann, "A parameter-adaptive PID-controller with stepwise parameter optimizalion," Automaticn, vol. 23, no. 4, pp. 449-45i, 1987.

[15] P. J. Parker and H. H. Bitmead. "Adaptive frequency response identification," in Procectings of the IEEE Conference on Decision and Confrol, pp. 348-353, Los Angeles, December 1987.

[16] M. Dentino, B. Widrow, and J. MrCool, "Adaptive filtering in the frequency domain," Procecdings of the IEEE, vol. 66, pp. 1658-1659, December 1978.

[17] A. Tzes and S. Yurkovich, "Application and comparison of on-line identification methods for flexible manipulator control," in Proc. International Conference on Advanced Robotics, Columbus, $\mathrm{OH}, 1989$. (to appear).

[18] L. Ljung, System Identification Theory For The IIser. Englewood Cliffs, N.J: Prentice-Hall, 1987.

[19] R. LaMaire, L. Valavani, M. Athans, and G. Stein, "A frequency-domain estimator for use in adaptive control systems," in Procedings of the American Control Conference, pp. 238-244, Minneapolis, MN, June 1987.

[20] A. Tzes and S. Yurkovich, "A new approach to frequency domain identification for flexible structure control," in Proceedings of the IEEE ('onference on Decision and Control, pp. 16271632, Austin, TX, December 1988.

[21] D. R. Brillinger, Time Series: Data Analysis and Theory. San Francisco: Holden Day, 1981. 


\section{ROBOTIC END-EFFECTORS AND HAND CONTROLLERS}


